\title{
Distributed fault detection and estimation in cyber-physical systems subject to actuator faults
}

\author{
Dezhi Xua ${ }^{\mathrm{a}}$, Fanglai Zhu ${ }^{\mathrm{b}, *}$, Zepeng Zhou ${ }^{\mathrm{b}}$, Xinggang Yan $^{\mathrm{c}}$ \\ ${ }^{a}$ School of Internet of Things Engineering, Jiangnan University, Wuxi, 214122, China \\ ${ }^{b}$ College of Electronics and Information Engineering, Tongji University, Shanghai 200092, China \\ ${ }^{c}$ School of Engineering and Digital Arts, University of Kent, Canterbury, CT2 7NT, U.K.
}

\begin{abstract}
The fault detection and estimation problems for the physical layer network in the cyber-physical systems with unknown external disturbances are investigated in this study. Both bias fault and loss of efficiency scenarios are considered for the actuators. Based on the adaptive threshold method and sliding mode observer approach, a distributed fault detection observer (DFDO) is constructed for each physical layer node to detect the occurrence of actuator faults. Then a relative global estimation error system is defined for the distributed fault estimation observer (DFEO). Compared with the existing results, the proposed DFEO can provide the estimation for not only the actuator bias faults but also the actuators' efficiency factors under the impact of exogenous disturbance with two gain dynamic update processes. Finally, the feasibility and effectiveness of the given DFDO and the DFEO are examined by Lyapunov stability method and the simulation results.
\end{abstract}

(C) 2011 Published by Elsevier Ltd.

Keywords: Cyber-physical system; cyber-physical system security; fault detection; fault estimation; adaptive sliding mode observer

\section{Introduction}

During the past few years, there have been considerable improvements of information technology, embedded systems and the distributed actuator/sensor networks. All these advances have been attracting the attentions on the development of the cyber-physical systems (CPSs) which are now utilized to power generation [1,2], water distribution systems [3] and other advanced complex systems. According to [4, 5], the integration of the CPSs usually involves: 1) physical or engineered systems which are often in large-scales, and 2) a cyber part that is based on communication networks and computational resources. The cyber part of the CPSs is available to monitor, coordinate and the corresponding part in the CPSs. The core of the CPS, which is different from the conventional complex system, is the emphasis on the physical systems' intelligence that is represented by the self-awareness, adaptiveness to the environment, and the automation of the physical systems [6].

The key challenges in the development of the CPSs are the reliability and the safety demands. Faults in the CPSs may lead to the malfunction of the whole system and some catastrophic consequences. In order to meet the safety and reliability demands, the CPSs should possess the ability of fault diagnosis and fault tolerance. During the past decades,

\footnotetext{
${ }^{*}$ Corresponding author

Email address: zhufanglai@tongji.edu.cn (Fanglai Zhu)
} 
many methods have been developed to deal with the increasing demands on the fault diagnosis with respect to actuator or sensor faults. According to the distributed fault detection method proposed in [6], the occurrence of the sensor fault can be monitored effectively in the sensor set for the physical part of the cyber superstratum. Based on a unified modeling framework, Pasqualetti et al. [7] proposed centralized and distributed fault detection and identification monitors for the CSPs with respect to the fundamental monitoring limitations. In [8], on the basis of local model and measurements, Teixeria et al. proposed a distributed fault isolation strategy for a large networked systems under model uncertainties which are caused by the edges between subsystems. According to the status information provided by the neighbor agents, a distributed adversarial observer is constructed in [9] for each agent to detect the faults which are caused by exogenous incipient adversaries with respect to time delays. For offsetting the actuator faults, Zhao et al. proposed a high-gain-observer based fault tolerant controller in [10] while the end-to-end communication rates approach is also taken into consideration. As is wildly implemented in traditional FDI methods, the Kalman filter is utilized in [11] to monitor the occurrence of sensor faults in real CPS mechanistic model with the limitation of the CUSUM test. In [12], by integrating the adaptive threshold technique, Zhang et al. developed an adaptive fault detection observer for a nonlinear systems while the fault isolation problem is also solved. By considering the distributed filter technique, Jiang et al. [13] proposed a fault detection approach for a nonlinear multi-agent system under the impact caused by network time delay.

However, most of the existing researches are focused on the detection and the isolation demands for actuator or sensor fault, and few of these methods could provide the estimated amplitude for the actuator or sensor fault[14]. Estimating the value of actuator/sensor faults instead of giving an alarm under the circumstance of actuator/sensor faults is an important part during the design process of an active fault tolerant controller. According to the dynamics of an individual node of a network, a centralized sliding mode observer is constructed in [15] at supervisory monitoring level and the fault is reconstructed from the equivalent output error injection signal. In [17], a distributed fault estimation approach integrating with the sliding mode observer technique is proposed by the implement of the equivalent output error injection with respect to the available measured information. However, in the aforementioned two methods, the upper bound of the faults should be given in advance and this limits the application of these two methods in actual industrial process. By creating a meaningful observable subsystem in [19], Menon et al. designed a robust fault estimation observer for the multi-agent systems. Based on $\mathcal{H}_{\infty}$ theory and the directed graph technique, the fault estimation problem is addressed with the design of a class of distributed fault estimation observer in [20] with adjustable parameter methods. In [18], by designing an intermediate estimator for each agent, the actuator fault is then reconstructed while the external disturbances is considered. Although the limitation about the pre-knowledge of the faults is relaxed in Zhang [20] and Zhu's [18] research, the Lyapunov stability proof processes indicate that both of the two fault estimation methods can only obtain a uniformly ultimately bounded result and the accuracy of the fault estimation is based on tuning the correlated parameters.

Motivated by the aforementioned reasons and researches, a novel robust distributed fault diagnosis approach is proposed for the physical layer network of the cyber-physical systems in this paper. Specifically, on the basis of the states from neighbor agents, local output measurements and cyber layer nodes, a DFDO is then constructed for each node in physical layer network of the CPSs under the influence of the exogenous disturbances. Then a novel adaptive DFEO is developed to estimate the actuator bias fault or the efficiency factors of the actuators. Compared with the existing researches about the fault diagnosis approaches of the CPSs, the main contributions of this study are as:

- According to the adaptive threshold approach, the robust DFDO can provide a more accurate fault alarm and the false alarm rate can be reduced. In addition, the sliding mode observer technique in this fault detection observer also increases the accuracy of fault detection with respect to external disturbance.

- Comparing to the existing research, such as [18], the robust DFEO in this paper for each physical layer node can guarantee an accurate fault estimation which means the estimation error can converge to the small region near the origin when time $t$ goes to infinity. Besides, this fault estimation observer can estimate not only the actuator bias fault but also the efficiency factors of the actuators simultaneously.

This paper is organized as: Section 2 presents some necessary preliminaries. The cyber layer network and the physical layer network of the CPSs with individual nodes are modeled in Section 3 while the problem formation is also presented in Section 3. Both the DFDO and the DFEO designs are given in Section 4, respectively. A numerical simulation example is demonstrated in Section 5 which is followed by a conclusion in Section 6. 


\section{Preliminaries}

An undirected graph $\mathcal{G}$ is a pair $\mathcal{G}=(\mathcal{V}, \mathcal{E})$ with a nonempty finite collection of $N$ nodes, namely $\mathcal{V}=(1, \ldots, N)$, and a set of edges $\mathcal{E} \subseteq \mathcal{V} \times \mathcal{V}$. For a certain edge $\left(v_{i}, v_{j}\right), v_{i}$ is a neighbor of $v_{j}$, therefore, $v_{j}$ is in the $i$ th node's neighbor set which can be denoted as $N_{i}=\{j:(j, i) \in \mathcal{E}\}$. In a undirected graph $\mathcal{G}$, the adjacency matrix $\mathcal{A}=\left[\alpha_{i j}\right]$ holds that $\alpha_{i j}=1$ if $(i, j) \in \mathcal{E}$ and $\alpha_{i j}=0$ otherwise while the Laplacian matrix $\mathcal{L} \in \mathcal{R}^{N \times N}$ is defined as $\mathcal{L}_{i j}=-a_{i j}$ where $i \neq j$ and $\mathcal{L}_{i i}=\Sigma_{i \neq j} \alpha_{i j}$.

Through out the paper, the following notations are defined; $\lambda_{\min (\max )}(\cdot)$ is the minimum (maximum) value of the matrix eigenvalue, $\operatorname{diag}\{\ldots\}$ represents a diagonal matrix and $\otimes$ is denoted as the Kronecker product of two matrices.

\section{Problem formation}

\subsection{Cyber-physical system dynamics}

From the control engineering aspect, the CPSs are the integration of physical processes and embedded computers with network communication $[5,6,21,22]$. Therefore, an actual CPSs can be modeled as the integration of physical layer network and cyber layer network where both of networks are interdependent.

Inspired by $[6,22,23]$, the CPSs is described as follows

$$
\begin{gathered}
\dot{x}_{i}(t)=\Omega\left(x_{i}(t)\right)+\sum_{j=1}^{N} a_{i j} \Gamma_{1} x_{j}(t)+\sum_{j=1}^{N} c_{i j} \Gamma_{3} y_{j}\left(t-\tau_{1}\right) \\
\dot{y}_{i}(t)=g\left(y_{i}(t)\right)+G u_{i}(t)+\sum_{j=1}^{N} b_{i j} \Gamma_{2} y_{j}(t)+\sum_{j=1}^{N} d_{i j} \Gamma_{4} x_{j}\left(t-\tau_{2}\right)+T \omega_{i}(t)
\end{gathered}
$$

where $x_{i}(t) \in R^{n}$ is the $i$ th $(1 \leq i \leq N)$ node's state of the cyber layer network, $y_{i}(t) \in R^{n}$ is the $i$ th $(1 \leq i \leq N)$ node's state of the physical layer network. $u_{i}(t) \in R^{m}$ is the control input which is utilized to generate proper control signal for the $i$ th physical layer network's node to synchronize with the $i$ th cyber layer network's node. $A=\left[a_{i j}\right]_{N \times N}$ is the topology structure of cyber layer network and $C=\left[c_{i j}\right]_{N \times N}$ indicates the topology structure between cyber layer network nodes and physical layer network nodes. Similarly, $B=\left[b_{i j}\right]_{N \times N}$ and $D=\left[d_{i j}\right]_{N \times N} . \Omega(\cdot)$ and $g(\cdot)$ are continuous nonlinear functions. $\Gamma_{1} \in R^{n \times n}$ and $\Gamma_{2} \in R^{n \times n}$ are the inner coupling matrix of the cyber layer network and the physical layer network respectively, $\Gamma_{3} \in R^{n \times n}$ is the outer coupling matrix of the cyber layer network, $\Gamma_{4} \in R^{n \times n}$ is the outer coupling matrix of the physical layer network, $\tau_{1}$ and $\tau_{2}$ denote fixed time-delays in the transmission of information between cyber layer network and physical layer network respectively, $\omega_{i}(t) \in R^{n}$ is the external disturbance in the actual environment. $G$ and $T$ are matrices with proper dimensions. In addition, the cyberphysical systems' structure is demonstrated in Fig. 1.

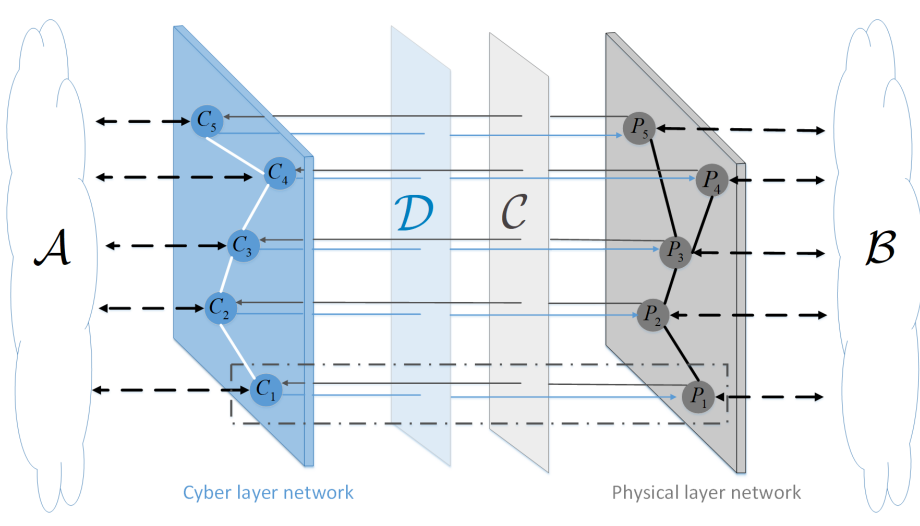

Figure 1: The structure of interconnected cyber-physical systems 


\subsection{Fault models}

Recently, there have been considerable interests in the fault diagnosis schemes for cyber-physical systems with respect to actuator faults and sensor faults [11,22]. Faults in the CPSs can lead to the performance degradation, and even disturb the physical property of the system as well as result in the malfunction of actual components. In this paper, only actuator faults, which take place on the physical layer nodes, are taken into consideration. Therefore, the faulty control input $u_{i}^{F}(t)$ can be modeled as

$$
u_{i}^{F}(t)=\Phi_{i} u_{i}(t)+f_{i}(t)
$$

where $\Phi_{i}=\operatorname{diag}\left\{\phi_{i 1}, \ldots, \phi_{i k}, \ldots, \phi_{i m}\right\}(k=1,2, \ldots, m)$ with $\phi_{i k} \in(0,1]$ is the effectiveness matrix with time invariant terms and $\phi_{i}$ is the so-called efficiency factor which is defined as a positive constant, $f_{i}(t)=\left[f_{i 1}, \ldots, f_{i k}, \ldots, f_{i m}\right]^{T}(k=$ $1,2, \ldots, m)$ is the bias fault input vector which is injected in the actuators in an additive form. The relations between actuator faults and the corresponding parameters are shown in Tab. 1

\begin{tabular}{lcc}
\hline \hline Fault Type & $\phi_{i k}$ & $f_{i k}$ \\
\hline Bias fault & 1 & $f_{i k} \neq 0$ \\
\hline Loss of effectiveness(LOE) & $0<\phi_{i k}<1$ & 0 \\
\hline
\end{tabular}

Table 1: Parameters for different types of actuator faults.

Hence, the physical layer node in cyber physical system with actuator faults is given as

$$
\dot{y}_{i}(t)=g\left(y_{i}(t)\right)+G \Phi_{i} u_{i}(t)+G f_{i}(t)+\sum_{j=1}^{N} b_{i j} \Gamma_{2} y_{j}(t)+\sum_{j=1}^{N} d_{i j} \Gamma_{4} x_{j}\left(t-\tau_{2}\right)+T \omega_{i}(t)
$$

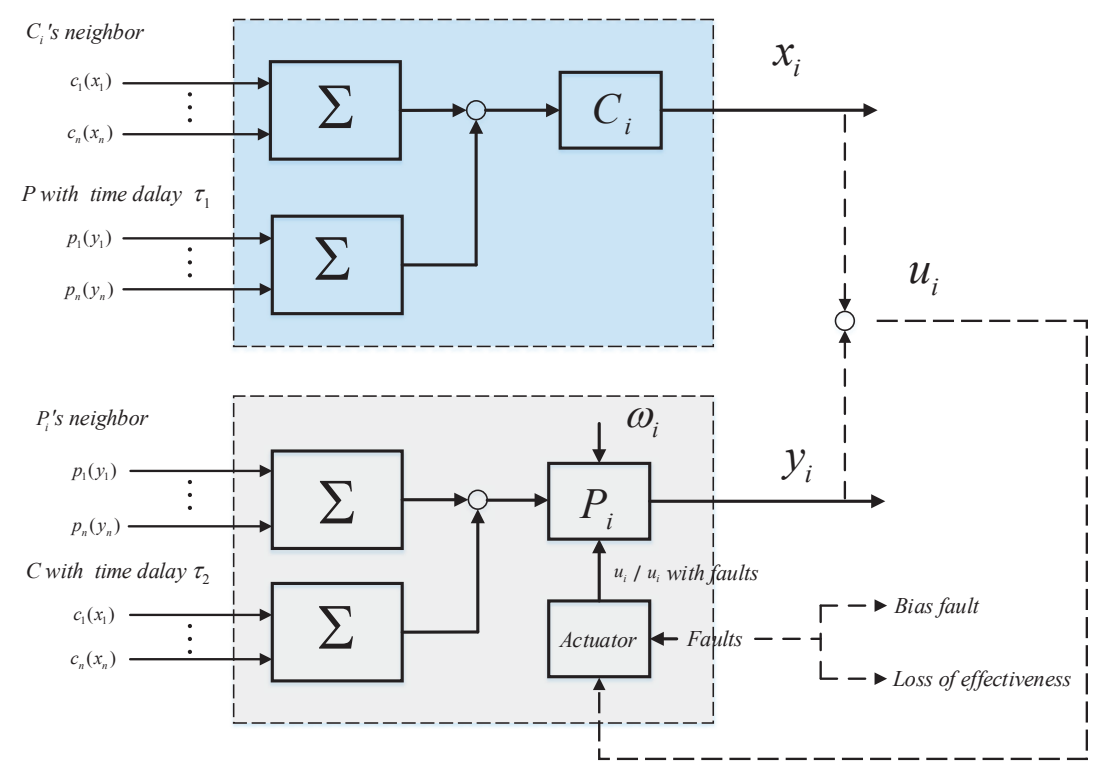

Figure 2: Description of the $i$ th CPS affected by actuator faults

\subsection{Problem formulation}

In this study, a novel actuator fault diagnose approach is investigated with respect to the CPSs in the following aspects: 
- For each physical layer node, designing a sliding mode DFDO on the basis of the local output and the state estimations from neighbors so as to detect actuator faults automatically and timely;

- An adaptive DFEO is constructed for each physical layer node so as to acquire the accurate actuator fault estimation and the states of the corresponding physical layer node.

For the development of the fault diagnosis scheme, the following assumptions are required:

Assumption 1. The cyber layer node's state $x_{i}(t)$ and the physical layer node's state $y_{i}(t)$ are observable.

Assumption 2. [15] It is assumed that for all $x, y \in R^{n}$, the function $g(x)$ is bounded with a Lipschitz constant $\varepsilon$ and holds that

$$
\|g(x)-g(y)\| \leq \varepsilon\|x-y\|
$$

Assumption 3. [28, 29] For each physical layer node, the external disturbance $\omega_{i}$ is bounded and satisfying $\left\|\omega_{i}\right\| \leq \bar{\omega}_{i}$, where $\bar{\omega}_{i}$ is a pre-known positive scalar.

Assumption 4. $[18,25]$ For each bias fault $f_{i}$, it holds that $\left\|f_{i}\right\| \leq \bar{f}_{i}$ and $\left\|\dot{f}_{i}(t)\right\|<\beta_{i}$ with $\beta_{i} \geq 0$. In addition, the bias fault considered here is slowly time-varying and $\beta_{i}$ is a rather small value.

Remark 1. As the actuator faults may take place in the operation of physical layer nodes, many existing researches, such as $[15,18]$, have dealt with this issue by investigating the bias fault only. In this paper, the detection and estimation for both LOE fault and bias fault are considered.

Remark 2. Assumption 4 indicates that the actuator faults have unknown boundaries whereas the sliding mode observers proposed in [15] and [17] require that the fault is bounded by a known function. In addition, the derivative of bias fault $f_{i}$, which will be investigated in the design of fault estimation observer, is bounded by a positive constant.

Remark 3. In this paper, the main concern is to develop an accurate actuator fault detection scheme and a corresponding actuator fault estimation strategy with respect to the cyber-physical systems. Therefore, based on the signal transmit structure proposed in [16], the states of the physical layer and the cyber layer are known to each other for simplicity.

\section{Main result}

In this section, the DFDO and the DFEO are proposed with sliding mode observer technique for physical layer nodes in cyber-physical systems. The DFDO with adaptive detection threshold is initially presented. Then, the adaptive sliding mode DFEO is utilized to estimate the effectiveness factors in the effective matrix and bias fault.

\subsection{Distributed fault detection observer design}

The DFDO for the $i$ th physical layer node is formulated as

$$
\begin{aligned}
\dot{\hat{y}}_{i}(t)=g\left(\hat{y}_{i}(t)\right) & +G u_{i}(t)+T \bar{\omega}_{i} \operatorname{sign}\left(y_{i}(t)-\hat{y}_{i}(t)\right)+H_{i}\left(y_{i}(t)-\hat{y}_{i}(t)\right) \\
& +\sum_{j=1}^{N} b_{i j} \Gamma_{2} y_{j}(t)+\sum_{j=1}^{N} d_{i j} \Gamma_{4} x_{j}\left(t-\tau_{2}\right)
\end{aligned}
$$

where $\hat{y}_{i}$ is the estimated state of the $i$ th physical layer node, $H_{i}=\operatorname{diag}\left\{h_{i 1}, \ldots, h_{i n}\right\}$ with $h_{i k}>0(k=1, \ldots, n)$ is the gain matrix for the observer. Subtracting (6) from (4), the $i$ th node's error dynamic equation is further obtained like

$$
\dot{\tilde{y}}_{i}=g\left(y_{i}(t)\right)-g\left(\hat{y}_{i}(t)\right)+G\left(\Phi_{i}-I_{m}\right) u_{i}(t)+G f_{i}(t)+T\left(\omega_{i}(t)-\bar{\omega}_{i} \operatorname{sign}\left(\tilde{y}_{i}(t)\right)\right)-H_{i} \tilde{y}_{i}(t)
$$

According to the proposed DFDO (6), one of the main results is firstly presented as, 
Theorem 1. For the faulty physical layer nodes (4) with actuator faults in cyber-physical systems, suppose that Assumptions 1-3 hold, if there exists a positive definite matrix $Q_{i}$ and a symmetric matrix $P_{i}=P_{i}^{T}>0$, such that

$$
Q_{i}=P_{i}\left(H_{i}-\varepsilon I_{n}\right)+\left(H_{i}-\varepsilon I_{n}\right)^{T} P_{i}-2 P_{i} P_{i}>0
$$

then the adaptive threshold for the $i$ th fault detection observer is given as

$$
\chi_{i}=\frac{\lambda_{\max }\left(P_{i}\right)}{\lambda_{\min }\left(P_{i}\right)} e^{-\left(\lambda_{\min }\left(Q_{i}\right) / \lambda_{\max }\left(P_{i}\right)\right)\left(t-t_{0}\right)}\left\|\tilde{y}_{i}\left(t_{0}\right)\right\|^{2}
$$

If the 2-norm of error $\tilde{y}_{i}$ exceeds the adaptive threshold $\sqrt{\chi_{i}}$, then it can be determined that at least one of the actuators in the $i$ th physical layer node is faulty.

Proof. Considering the following Lyapunov candidate for the $i$ th physical layer node

$$
V_{d i}=\tilde{y}_{i}^{T} P_{i} \tilde{y}_{i}
$$

In actuator healthy case: In this case, there is no fault taking place on the $i$ th physical layer node's actuators, which means that $\Phi_{i}=I_{m}$ and $f_{i}=0$. The time derivative of $V_{d i}$ is as

$$
\begin{aligned}
\dot{V}_{d i}= & \left.2 \tilde{y}_{i}^{T} P_{i}\left(g\left(y_{i}(t)\right)-g\left(\hat{y}_{i}(t)\right)-H_{i} \tilde{y}_{i}(t)\right)\right) \\
& +2 \tilde{y}_{i}^{T} P_{i} T\left(\omega_{i}(t)-\bar{\omega}_{i} \operatorname{sign}\left(\tilde{y}_{i}(t)\right)\right)
\end{aligned}
$$

According to Assumption 2, it can be obtained that

$$
\dot{V}_{d i} \leq-2 \tilde{y}_{i}^{T} P_{i}\left(H_{i}-\varepsilon I_{n}\right) \tilde{y}_{i}(t)
$$

It should be found that

$$
P_{i}\left(H_{i}-\varepsilon I_{n}\right)+\left(H_{i}-\varepsilon I_{n}\right)^{T} P_{i}>P_{i}\left(H_{i}-\varepsilon I_{n}\right)+\left(H_{i}-\varepsilon I_{n}\right)^{T} P_{i}-2 P_{i} P_{i}=Q>0
$$

Therefore, the derivation of $V_{d i}$ satisfies

$$
V_{d i} \leq-\tilde{y}_{i}^{T} Q \tilde{y}_{i}<0
$$

In addition, it can be noted from (9) that

$$
\lambda_{\min }\left(P_{i}\right)\left\|\tilde{y}_{i}\right\|^{2} \leq V_{d i} \leq \lambda_{\max }\left(P_{i}\right)\left\|\tilde{y}_{i}\right\|^{2}
$$

Therefore, the following inequality can be obtained further

$$
\dot{V}_{d i} \leq-\frac{\lambda_{\min }\left(Q_{i}\right)}{\lambda_{\max }\left(P_{i}\right)} V_{d i}
$$

According to (13), the error signal $\left\|\tilde{y}_{i}\right\|^{2}$ satisfies the following formula when there is no actuator fault

$$
\left\|\tilde{y}_{i}\right\|^{2} \leq \frac{\lambda_{\max }\left(P_{i}\right)}{\lambda_{\min }\left(P_{i}\right)} e^{-\left(\lambda_{\min }\left(Q_{i}\right) / \lambda_{\max }\left(P_{i}\right)\right)\left(t-t_{0}\right)}\left\|\tilde{y}_{i}\left(t_{0}\right)\right\|^{2} \triangleq \chi_{i}
$$

In actuator faulty case: In this case, the fault takes place on the $i$ th physical layer node's actuators, which means that $\Phi_{i} \neq I_{m}$ or $f_{i} \neq 0$. we have

$$
\begin{aligned}
& \dot{V}_{d i} \leq-2 \tilde{y}_{i}^{T} P_{i}\left(H_{i}-\varepsilon I_{n}\right) \tilde{y}_{i}(t)+2 \tilde{y}_{i}^{T} P_{i} G\left(\Phi_{i}-I_{m}\right) u_{i}(t)+2 \tilde{y}_{i}^{T} P_{i} G f_{i}(t) \\
& \leq-\tilde{y}_{i}^{T} Q_{i} \tilde{y}_{i}+\left[G\left(\Phi_{i}-I_{m}\right) u_{i}(t)\right]^{T}\left[G\left(\Phi_{i}-I_{m}\right) u_{i}(t)\right]+\left[G f_{i}(t)\right]^{T}\left[G f_{i}(t)\right]
\end{aligned}
$$

Noticing the inequality in (12), it follows that

$$
\left\|\tilde{y}_{i}\right\|^{2} \leq \frac{\lambda_{\max }\left(P_{i}\right)}{\lambda_{\min }\left(P_{i}\right)} e^{-\left(\lambda_{\min }\left(Q_{i}\right) / \lambda_{\max }\left(P_{i}\right)\right)\left(t-t_{0}\right)}\left\|\tilde{y}_{i}\left(t_{0}\right)\right\|^{2}
$$




$$
\begin{gathered}
+\frac{1}{\lambda_{\min }\left(P_{i}\right)} \int_{t_{0}}^{t} e^{-\left(\lambda_{\min }\left(Q_{i}\right) / \lambda_{\max }\left(P_{i}\right)\right)(t-\tau)} \\
\times\left\{\left[G\left(\Phi_{i}-I_{m}\right) u_{i}(\tau)\right]^{T}\left[G\left(\Phi_{i}-I_{m}\right) u_{i}(\tau)\right]+\left[G f_{i}(\tau)\right]^{T}\left[G f_{i}(\tau)\right]\right\} d \tau
\end{gathered}
$$

The above inequality indicates that $\left\|\tilde{y}_{i}\right\|$ will exceed the adaptive threshold $\chi_{i}$ once there exist bias faults or loss of effectiveness faults in actuators. As a result, the actuator faults can be detected timely and accurately.

Remark 4. Different from the fault detection method proposed in $[8,26]$, the DFDO proposed in this paper provides a more accurate way to detect the actuator faults by utilizing the proposed adaptive threshold, whereas fault detection observers developed in $[8,26]$ are based on fixed thresholds which are derived from experience in advance. This merit is owing to the decreasing factor $e^{-\left(\lambda_{\min }\left(Q_{i}\right) / \lambda_{\max }\left(P_{i}\right)\right)\left(t-t_{0}\right)}$ which can provide an exponentially decrease for the time response in the adaptive fault detection threshold. Therefore, the proposed DFDO possess a lower false missing rate compared to the ones in $[8,26]$.

Remark 5. With the implementation of sliding mode observer technique, the fault detection observer in this paper shows a better robustness performance to the external disturbance in actual environment when compared to [8,26].

Remark 6. To suppress the chattering phenomenon caused by the sign function, the discontinuous function $\operatorname{sign}\left(\tilde{y}_{i}\right)$ can be replaced by $\operatorname{sat}\left(\tilde{y}_{i} / \epsilon_{i}\right)$ where $\epsilon_{i}$ is the boundary thickness parameter of the saturation function.

\subsection{Distributed fault estimation observer design}

Recall the problem of distributed fault estimation presented in 3.3. The distributed fault estimation observer proposed here for the $i$ th physical layer node is utilized to estimated the efficiency factors and the bias faults on-line. Before giving the formulation of the distributed fault estimation observer, there are two new variables that need to be defined here

$$
\begin{gathered}
\phi_{i}=\left[\phi_{i 1}, \ldots, \phi_{i m}\right]^{T} \\
U_{i}=\operatorname{diag}\left\{u_{i 1}, \ldots, u_{i m}\right\}
\end{gathered}
$$

Consequently, the faulty dynamic of the physical layer nodes with actuator fault can be rewritten as follows

$$
\dot{y}_{i}(t)=g\left(y_{i}(t)\right)+G U_{i}(t) \phi_{i}+G f_{i}(t)+\sum_{j=1}^{N} b_{i j} \Gamma_{2} y_{j}(t)+\sum_{j=1}^{N} d_{i j} \Gamma_{4} x_{j}\left(t-\tau_{2}\right)+T \omega_{i}(t)
$$

By noting (17), the DFEO for the $i$ th node with actuator fault is presented as follows

$$
\begin{aligned}
\dot{\hat{y}}_{i}(t)=g\left(\hat{y}_{i}(t)\right) & +\sum_{j=1}^{N} b_{i j} \Gamma_{2} \hat{y}_{j}(t)+\sum_{j=1}^{N} d_{i j} \Gamma_{4} x_{j}\left(t-\tau_{2}\right)+G U_{i}(t) \hat{\phi}_{i}+G \hat{f}_{i}(t) \\
& +H_{e i}\left(y_{i}(t)-\hat{y}_{i}(t)\right)+T \bar{\omega}_{i} \operatorname{sign}\left(y_{i}(t)-\hat{y}_{i}(t)\right)
\end{aligned}
$$

The adaptive updating algorithms for $\hat{\phi}_{i}$ and $\hat{f}$ are given as

$$
\begin{aligned}
\dot{\hat{\phi}}_{i} & =-\mu_{i 1} \hat{\phi}_{i}+\frac{1}{l_{i 1}} U_{i}^{T} G^{T} \tilde{y}_{i} \\
\dot{\mu}_{i 1} & =-v_{i 1} \mu_{i 1}, \quad \mu_{i 1}(0)>0 \\
\dot{\hat{f}}_{i} & =-\mu_{i 2} \hat{f}_{i}+\frac{1}{l_{i 2}} G^{T} \tilde{y}_{i} \\
\dot{\mu}_{i 2} & =-v_{i 2} \mu_{i 2}, \quad \mu_{i 2}(0)>0
\end{aligned}
$$

where $l_{i k}>0$ and $v_{i k}>0(k=1,2)$. Therefore, the overall dynamics of the proposed distributed fault estimation observer can be formulated by

$$
\dot{\hat{y}}=g(\hat{y}(t))+\left(B \otimes \Gamma_{2}\right) \hat{y}+\left(D \otimes \Gamma_{4}\right) x\left(t-\tau_{2}\right)+\left(I_{N} \otimes G\right) U(t) \hat{\phi}+\left(I_{N} \otimes G\right) \hat{f}(t)
$$




$$
+H_{e}(y(t)-\hat{y}(t))+\left(I_{N} \otimes T\right)\left[\begin{array}{c}
\bar{\omega}_{1} \operatorname{sign}\left(\tilde{y}_{1}\right) \\
\vdots \\
\bar{\omega}_{N} \operatorname{sign}\left(\tilde{y}_{N}\right)
\end{array}\right]
$$

where $\hat{y}(t)=\left[\hat{y}_{1}^{T}(t), \ldots, \hat{y}_{N}^{T}(t)\right]^{T}, x\left(t-\tau_{2}\right)=\left[x_{1}^{T}\left(t-\tau_{2}\right), \ldots, x_{N}^{T}\left(t-\tau_{2}\right)\right]^{T}, g(\hat{y}(t))=\left[g\left(\hat{y}_{1}(t)\right)^{T}, \ldots, g\left(\hat{y}_{N}(t)\right)^{T}\right]^{T}, U(t)=$ $\operatorname{diag}\left\{U_{1}(t), \ldots, U_{N}(t)\right\}, \hat{\phi}=\left[\hat{\phi}_{1}^{T}, \ldots, \hat{\phi}_{N}^{T}\right]^{T}, \hat{f}=\left[\hat{f}_{1}^{T}(t), \ldots, \hat{f}_{N}^{T}(t)\right]^{T}$ and $H_{e}=\operatorname{diag}\left\{H_{e 1}, \ldots, H_{e N}\right\}$. In addition, based on (4), the overall dynamics of the physical layer nodes can be represented by

$$
\dot{y}(t)=g(y(t))+\left(B \otimes \Gamma_{2}\right) y(t)+\left(D \otimes \Gamma_{4}\right) x\left(t-\tau_{2}\right)+\left(I_{N} \otimes G\right) U(t) \phi+\left(I_{N} \otimes G\right) f(t)+\left(I_{N} \otimes T\right)\left[\begin{array}{c}
\omega_{1}(t) \\
\vdots \\
\omega_{N}(t)
\end{array}\right]
$$

where $y(t)=\left[y_{1}(t), \ldots, y_{N}(t)\right]^{T}, \phi=\left[\phi_{1}, \ldots, \phi_{N}\right]^{T}$, and $f(t)=\left[f_{1}^{T}(t), \ldots, f_{N}^{T}(t)\right]^{T}$. Denoting $\tilde{y}(t)=y(t)-\hat{y}(t)$, $\tilde{\phi}=\phi-\hat{\phi}, \tilde{f}=f-\hat{f}$. The error dynamic equation between (23) and (24) can be represented as follows

$$
\dot{\tilde{y}}=g(y(t))-g(\hat{y}(t))+\left(B \otimes \Gamma_{2}\right) \tilde{y}+\left(I_{N} \otimes G\right) U(t) \tilde{\phi}+\left(I_{N} \otimes G\right) \tilde{f}-H_{e} \tilde{y}+\left(I_{N} \otimes T\right)\left\{\left[\begin{array}{c}
\omega_{1}(t) \\
\vdots \\
\omega_{N}(t)
\end{array}\right]-\left[\begin{array}{c}
\bar{\omega}_{1} \operatorname{sign}\left(\tilde{y}_{1}\right) \\
\vdots \\
\bar{\omega}_{N} \operatorname{sign}\left(\tilde{y}_{N}\right)
\end{array}\right]\right\}
$$

Based on (23), (24) and (25), the second main result of this paper is ready to be presented below.

Theorem 2. If Assumptions 1-5 hold, then the DFEO (18) is designed for each physical layer node with actuator bias fault or LOE fault. The error dynamic equation (25) is asymptotically stable by selecting proper $H_{e i}$, such that the inequality $\lambda_{\min }\left(H_{e}\right)-\lambda_{\max }\left(B \otimes \Gamma_{2}\right)-\varepsilon>0$ holds, then the adaptive DFEO can offer an accurate estimation of actuator faults.

Proof. Here, the Lyapunov function is as

$$
\begin{gathered}
V=\frac{1}{2} \tilde{y}^{T} \tilde{y}+\sum_{i=1}^{N} \frac{l_{i 1}}{2 v_{i 1}} \phi_{i}^{T} \phi_{i} \mu_{i 1}+\sum_{i=1}^{N} \frac{l_{i 2}}{2 v_{i 2}} f_{i}^{T} f_{i} \mu_{i 2} \\
+\frac{1}{2} \tilde{\phi}^{T}\left[\begin{array}{ccc}
l_{11} I_{m} & & \\
& \ddots & \\
& & l_{N 1} I_{m}
\end{array}\right] \tilde{\phi}+\frac{1}{2} \tilde{f}^{T}\left[\begin{array}{ccc}
l_{12} I_{m} & & \\
& \ddots & \\
& & l_{N 2} I_{m}
\end{array}\right] \tilde{f}
\end{gathered}
$$

As for $\dot{V}$, it holds

$$
\begin{aligned}
& \dot{V}=\tilde{y}^{T}\left(g(y(t))-g(\hat{y}(t))+\left(B \otimes \Gamma_{2}\right) \tilde{y}+\left(I_{N} \otimes G\right) \tilde{f}+\left(I_{N} \otimes G\right) U(t) \tilde{\phi}-H_{e} \tilde{y}\right) \\
& +\tilde{y}^{T}\left(I_{N} \otimes T\right)\left[\begin{array}{c}
\omega_{1}(t)-\bar{\omega}_{1} \operatorname{sign}\left(\tilde{y}_{1}\right) \\
\vdots \\
\omega_{N}(t)-\bar{\omega}_{N} \operatorname{sign}\left(\tilde{y}_{N}\right)
\end{array}\right]+\sum_{i=1}^{N} \frac{l_{i 1}}{2 v_{i 1}} \phi_{i}^{T} \phi_{i} \dot{\mu}_{i 1}+\sum_{i=1}^{N} \frac{l_{i 2}}{2 v_{i 2}} f_{i}^{T} f_{i} \dot{\mu}_{i 2} \\
& +\tilde{f}^{T}\left[\begin{array}{ccc}
l_{12} I_{m} & & \\
& \ddots & \\
& & l_{N 2} I_{m}
\end{array}\right] \dot{f}+\left[\tilde{f}_{1}^{T}, \ldots, \tilde{f}_{N}^{T}\right]\left[\begin{array}{ccc}
l_{12} I_{m} & & \\
& \ddots & \\
& & l_{N 2} I_{m}
\end{array}\right]\left[\begin{array}{c}
\mu_{12} \hat{f}_{1}-\frac{1}{l_{12}} G^{T} \tilde{y}_{1} \\
\vdots \\
\mu_{N 2} \hat{f}_{N}-\frac{1}{l_{N 2}} G^{T} \tilde{y}_{N}
\end{array}\right] \\
& +\left[\tilde{\phi}_{1}^{T}, \ldots, \tilde{\phi}_{N}^{T}\right]\left[\begin{array}{lll}
l_{11} I_{m} & & \\
& \ddots & \\
& & l_{N 1} I_{m}
\end{array}\right]\left[\begin{array}{c}
\mu_{11} \hat{\phi}_{1}-\frac{1}{l_{11}} U_{1}^{T}(t) G^{T} \tilde{y}_{1} \\
\vdots \\
\mu_{N 1} \hat{\phi}_{N}-\frac{1}{l_{N 1}} U_{N}^{T}(t) G^{T} \tilde{y}_{N}
\end{array}\right]
\end{aligned}
$$


According to Assumptions 2-3, it follows that

$$
\begin{aligned}
& \dot{V} \leq \varepsilon\|\tilde{y}\|^{2}+\tilde{y}^{T}\left(B \otimes \Gamma_{2}\right) \tilde{y}+\tilde{y}^{T}\left(I_{N} \otimes G\right) \tilde{f}+\tilde{y}^{T}\left(I_{N} \otimes G\right) U(t) \tilde{\phi}-\tilde{y}^{T} H_{e} \tilde{y} \\
& +\tilde{f}^{T}\left[\begin{array}{ccc}
l_{12} I_{m} & & \\
& \ddots & \\
& & l_{N 2} I_{m}
\end{array}\right] \dot{f}-\tilde{f}^{T}\left[\begin{array}{ccc}
l_{12} I_{m} & & \\
& \ddots & \\
& & l_{N 2} I_{m}
\end{array}\right]\left[\begin{array}{c}
\frac{1}{l_{12}} G^{T} \tilde{y}_{1} \\
\vdots \\
\frac{1}{l_{N 2}} G^{T} \tilde{y}_{N}
\end{array}\right]+\tilde{f}^{T}\left[\begin{array}{ccc}
l_{12} \mu_{12} I_{m} & & \\
& \ddots & \\
& & l_{N 2} \mu_{N 2} I_{m}
\end{array}\right] \hat{f} \\
& +\tilde{\phi}^{T}\left[\begin{array}{lll}
l_{11} \mu_{11} I_{m} & & \\
& \ddots & \\
& & l_{N 1} \mu_{N 1} I_{m}
\end{array}\right]\left[\begin{array}{c}
\hat{\phi}_{1} \\
\vdots \\
\hat{\phi}_{N}
\end{array}\right]-\tilde{\phi}^{T}\left[\begin{array}{ccc}
l_{11} I_{m} & & \\
& \ddots & \\
& & l_{N 1} I_{m}
\end{array}\right]\left[\begin{array}{c}
\frac{1}{l_{11}} U_{1}^{T}(t) G^{T} \tilde{y}_{1} \\
\vdots \\
\frac{1}{l_{N 1}} U_{N}^{T}(t) G^{T} \tilde{y}_{N}
\end{array}\right] \\
& +\sum_{i=1}^{N} \frac{l_{i 1}}{2 v_{i 1}} \phi_{i}^{T} \phi_{i} \dot{\mu}_{i 1}+\sum_{i=1}^{N} \frac{l_{i 2}}{2 v_{i 2}} f_{i}^{T} f_{i} \dot{\mu}_{i 2} \\
& \dot{V} \leq\left(-\lambda_{\min }\left(H_{e}\right)+\lambda_{\max }\left(B \otimes \Gamma_{2}\right)+\varepsilon\right)\|\tilde{y}\|^{2}+\sum_{i=1}^{N} \frac{l_{i 1}}{2 v_{i 1}} \phi_{i}^{T} \phi_{i}\left(-v_{i 1} \mu_{i 1}\right)+\sum_{i=1}^{N} \frac{l_{i 2}}{2 v_{i 2}} f_{i}^{T} f_{i}\left(-v_{i 2} \mu_{i 2}\right) \\
& +\tilde{f}^{T}\left[\begin{array}{ccc}
l_{12} I_{m} & & \\
& \ddots & \\
& & l_{N 2} I_{m}
\end{array}\right] \dot{f}+\tilde{f}^{T}\left[\begin{array}{lll}
l_{12} \mu_{12} I_{m} & & \\
& \ddots & \\
& & l_{N 2} \mu_{N 2} I_{m}
\end{array}\right] \hat{f}+\tilde{\phi}^{T}\left[\begin{array}{lll}
l_{11} \mu_{11} I_{m} & & \\
& \ddots & \\
& & l_{N 1} \mu_{N 1} I_{m}
\end{array}\right] \hat{\phi} \\
& \leq\left(-\lambda_{\min }\left(H_{e}\right)+\lambda_{\max }\left(B \otimes \Gamma_{2}\right)+\varepsilon\right)\|\tilde{y}\|^{2}+\sum_{i=1}^{N} l_{i 2} \tilde{f}_{i} \dot{f}_{i}+\sum_{i=1}^{N} l_{i 2} \mu_{i 2} \tilde{f}_{i}^{T} \hat{f}_{i}+\sum_{i=1}^{N} l_{i 1} \mu_{i 1} \tilde{\phi}_{i}^{T} \hat{\phi}_{i} \\
& -\sum_{i=1}^{N} \frac{l_{i 1}}{2} \phi_{i}^{T} \phi_{i} \mu_{i 1}-\sum_{i=1}^{N} \frac{l_{i 2}}{2} f_{i}^{T} f_{i} \mu_{i 2}
\end{aligned}
$$

Noting that

$$
\begin{aligned}
& l_{i 1} \mu_{i 1} \tilde{\phi}_{i}^{T} \hat{\phi}_{i} \leq-\frac{1}{2} l_{i 1} \mu_{i 1} \tilde{\phi}_{i}^{T} \tilde{\phi}_{i}+\frac{1}{2} l_{i 1} \mu_{i 1} \phi_{i}^{T} \phi_{i} \\
& l_{i 2} \mu_{i 2} \tilde{f}_{i}^{T} \hat{f}_{i} \leq-\frac{1}{2} l_{i 2} \mu_{i 2} \tilde{f}_{i}^{T} \tilde{f}_{i}+\frac{1}{2} l_{i 2} \mu_{i 2} f_{i}^{T} f_{i}
\end{aligned}
$$

Therefore, it can be obtained that

$$
\dot{V} \leq\left(-\lambda_{\min }\left(H_{e}\right)+\lambda_{\max }\left(B \otimes \Gamma_{2}\right)+\varepsilon\right)\|\tilde{y}\|^{2}-\sum_{i=1}^{N} \frac{1}{2} l_{i 1} \mu_{i 1} \tilde{\phi}_{i}^{T} \tilde{\phi}_{i}-\sum_{i=1}^{N} \frac{1}{2} l_{i 2} \mu_{i 2} \tilde{f}_{i}^{T} \tilde{f}_{i}+\sum_{i=1}^{N} l_{i 2} \tilde{f}_{i}^{T} \dot{f}_{i}
$$

According to $[25,30]$ and Assumption 4, the above inequality indicates that the estimation error satisfy the ultimately uniformly bounded property by selecting the appropriate values of $H_{e i}, l_{i 1}, l_{i 2}, \mu_{i 1}$ and $\mu_{i 2}$. In addition, the estimation error can be eliminated when the actuator is confronted with constant bias faults or a slowly time-changing bias fault where $\dot{f}_{i}=0$ under this circumstance.

Remark 7. Compared with the DFEO in [18], the proposed adaptive DFEO can guarantee the asymptotical stability of the error dynamic system (25) when the actuator is suffering from a constant bias fault or a slowly time-changing bias fault, whereas the error dynamic system in [18] is only proved to be uniformly ultimately bounded no matter the bias fault is a constant or not. Therefore, the DFEO in this paper provides a better and more accurate estimation value of the actuator faults. 
Remark 8. It should be noted that in [24] only LOE fault is taken into consideration. In addition, the implementation of the DFEO in [18] is limited to the actuator bias fault case. However, the DFEO in this paper can tackle the estimation for both actuator bias fault and LOE fault simultaneously. Besides that, the fault estimation observers in $[18,24]$ focus on the linear systems whereas the DFEO in (18) not only can be used to linear system, but also can be implemented to nonlinear system.

Remark 9. There are two significant modifications in the adaptive algorithms of the DFEO, namely, two new dynamic processes of $\mu_{i 1}$ and $\mu_{i 2}$ in (20) and (22), which can result in two merits: i) It improves the accuracy and rapidity of the actuator fault estimation effectively; ii) The asymptotical stability of the error system (25) can be proved with respect to the existence of (20) and (22) when the constant bias fault or a slowly time-changing bias fault occurs.

Remark 10. The proposed Lyapunov function in (26) which is inherited from the traditional adaptive sliding mode observer actually can reflect the stability criteria of the presented DFEO. The time-varying terms $\sum_{i=1}^{N} \frac{l_{i 1}}{2 v_{i 1}} \phi_{i}^{T} \phi_{i} \mu_{i 1}$ and $\sum_{i=1}^{N} \frac{l_{i 2}}{2 v_{i 2}} f_{i}^{T} f_{i} \mu_{i 2}$ can converges to 0 due to the dynamic processes of $\mu_{i 1}$ and $\mu_{i 2}$ no matter the the actuator is faulty or not. Therefore, once the actuators are free from actuator faults, the estimation error will converge to zero while the stability of the fault free case is guaranteed by the deduction of the derivation for $V$ which is similar to the actuator faulty case.

\section{Simulation}

In this section, two different cases with two types of actuator faults have been taken into consideration. The effectiveness of the proposed DFDO and DFEO is to be demonstrated in this section. The cyber layer nodes and the physical layer nodes are represented by the Lorenz system. In addition, there are three nodes in the cyber layer network and the physical layer network respectively and the topology of the CPSs for each layer is shown in Fig.3.

The dynamic equations of the CPSs are given as follows

$$
\begin{gathered}
\dot{x}_{i}(t)=\Omega\left(x_{i}(t)\right)+\sum_{j=1}^{N} a_{i j} \Gamma_{1} x_{j}(t)+\sum_{j=1}^{N} c_{i j} \Gamma_{3} y_{j}\left(t-\tau_{1}\right) \\
\dot{y}_{i}(t)=g\left(y_{i}(t)\right)+G u_{i}(t)+\sum_{j=1}^{N} b_{i j} \Gamma_{2} y_{j}(t)+\sum_{j=1}^{N} d_{i j} \Gamma_{4} x_{j}\left(t-\tau_{2}\right)+T \omega_{i}(t)
\end{gathered}
$$

where $\Gamma_{1}=\Gamma_{2}=\Gamma_{3}=\Gamma_{4}=\operatorname{diag}\{1,1,1\}, T=I_{3}$, the time fixed delay are $\tau_{1}=\tau_{2}=0.5 s, A=B=C=D=$ $\left[\begin{array}{ccc}-2 & 1 & 1 \\ 1 & -2 & 1 \\ 1 & 1 & -2\end{array}\right]$, the distribution matrix $G=\left[\begin{array}{ccccc}1 & 0 & 0 & 1 / 2 & 1 / 3 \\ 0 & 1 & 0 & 1 / 2 & 1 / 3 \\ 0 & 0 & 1 & 1 / 2 & 1 / 3\end{array}\right]$ and the nonlinear functions $\Omega(\cdot)$ and $y(\cdot)$ in cyber layer nodes and physical layer nodes are given as

$$
\begin{gathered}
\Omega\left(x_{i}(t)\right)=\left[10 x_{i 2}-10 x_{i 1}, 28 x_{i 1}-x_{i 1} x_{i 3}-x_{i 2}, x_{i 1} x_{i 2}-8 / 3 x_{i 3}\right]^{T}, \quad i=1,2,3 \\
g\left(y_{i}(t)\right)=\left[10 y_{i 2}-10 y_{i 1}, 28 y_{i 1}-y_{i 1} y_{i 3}-y_{i 2}, y_{i 1} y_{i 2}-8 / 3 y_{i 3}\right]^{T}, \quad i=1,2,3 \\
\omega_{i}(t)=\left[\begin{array}{c}
1.5 \times 10^{-2}(3 \cos (t)+1) \\
2 \times 10^{-2}(1.5 \cos (t)+2 \sin (t)) \\
1.25 \times 10^{-2}(\cos (t)+2)
\end{array}\right], \quad i=1,2,3
\end{gathered}
$$

The initial states of the physical layer nodes and the cyber layer nodes are selected as $x_{1}(0)=[0.1,0.3,-0.1]^{T}$, $x_{2}(0)=[0.9,-0.7,0.3]^{T}, x_{3}(0)=[-0.2,0.5,0.1]^{T}, y_{1}(0)=[-0.3,0.5,0.3]^{T}, y_{2}(0)=[0.5,-0.3,0.5]^{T}$ and $y_{3}(0)=$ $[0.2,0.1,-0.3]^{T}$. Denoting $e_{i}(t)=y_{i}(t)-x_{i}(t)$ as the error between the corresponding cyber layer nodes and the physical layer nodes, the time response of the error in actuator fault free case is demonstrated in Fig. 4. It can be found that the error between the cyber layer nodes and the corresponding physical layer nodes can converge to 0 with the implementation of the basic control law proposed in [27] by integrating time delay control methods. In addition, from Figs. 4, 5 and 6, it can be noticed that all the states of the cyber layer nodes and the physical layer nodes are 


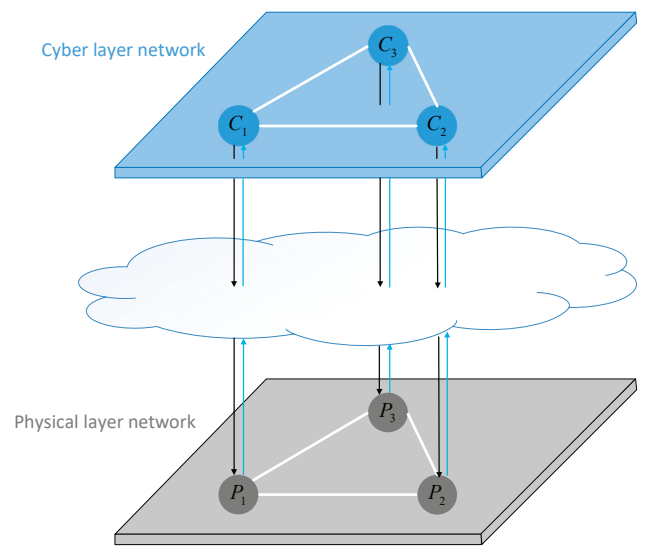

Figure 3: The topology structure of the CPSs in simulation
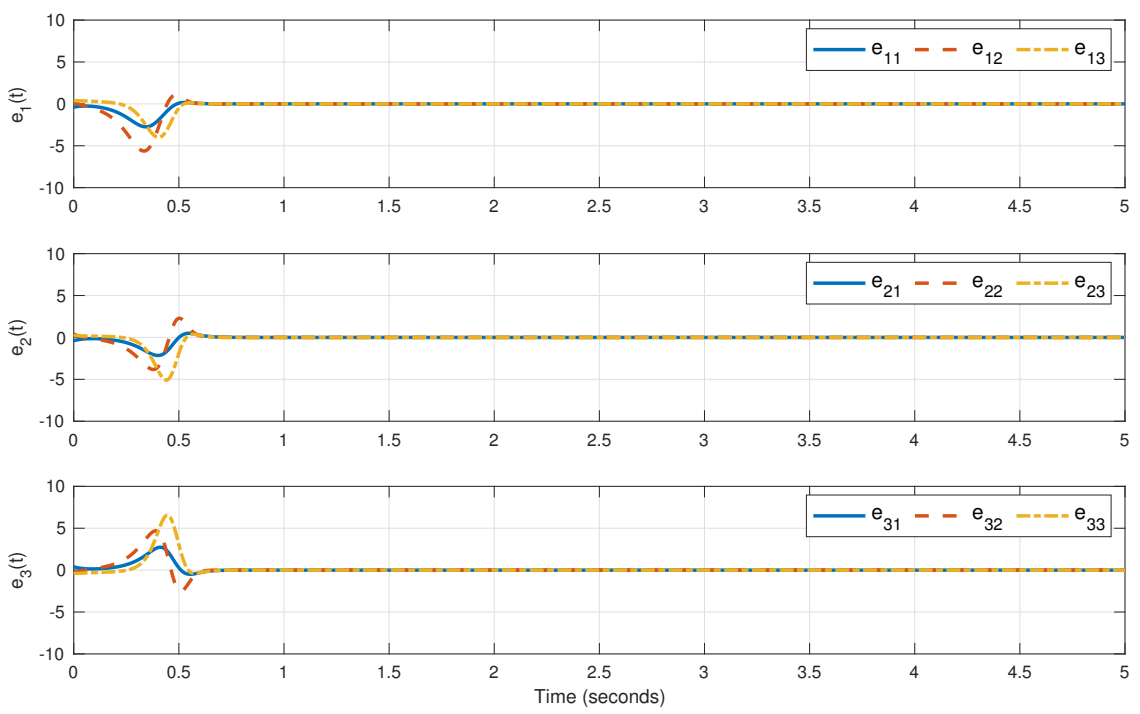

Figure 4: The time responses of state errors between the cyber layer nodes and the physical layer nodes(no fault) 

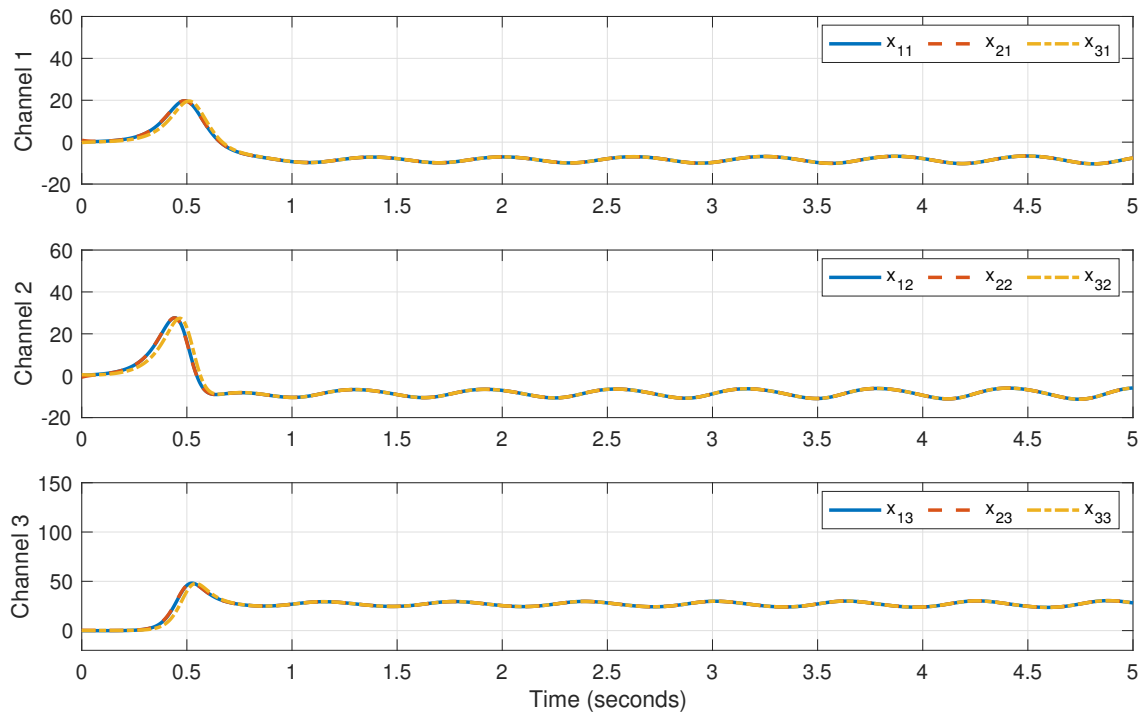

Figure 5: Time responses of the cyber layer nodes' states (no fault)
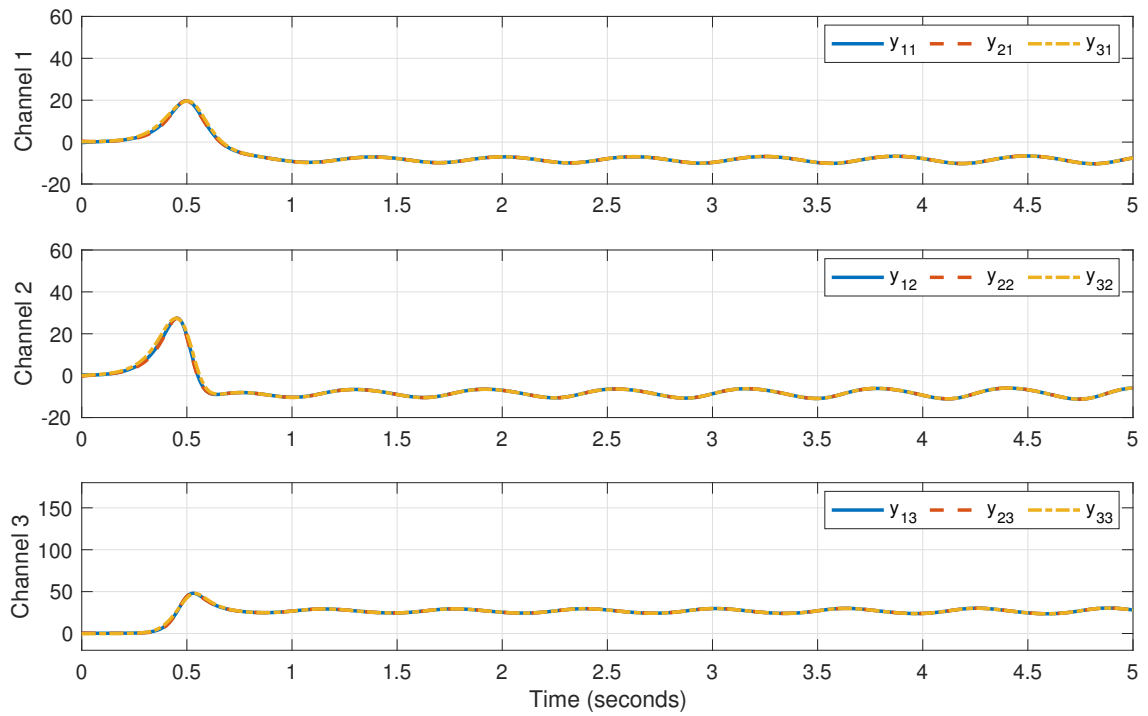

Figure 6: Time responses of the physical layer nodes' states (no fault) 
synchronized with the utilization of the aforementioned control law under the influence of the external disturbances acting on the physical layer nodes.

In order to verify the effectiveness of the proposed DFDO and the DFEO, two different actuator faulty cases are taken into consideration in the following subsections.

\subsection{Actuators with single type of fault}

In this subsection, the actuators in physical layer nodes are assumed to be affected by only one type of fault, namely, actuator bias fault. It is assumed that $y_{1}$ in the physical layer network is suffering from actuator bias fault and the bias fault vector is selected as

$$
f_{1}= \begin{cases}{[0,0,0,0,0]^{T},} & \text { if } 0 \leq t<1 s \\ {[20,0,0,0,0]^{T},} & \text { else }\end{cases}
$$

Noting that the bias fault takes place on the first actuator in $y_{1}$ at the first second. Here, the parameters of the DFDO and the DFEO are selected as $P_{i}=\operatorname{diag}\{1.2,1,1.3\}, H_{i}=\operatorname{diag}\{20,20,20\}$, the initial states of the fault detection observer are $\hat{y}_{d i}(0)=[-0.3,0.5,0.3]^{T}$. As for the fault estimation observer, the related parameters are given as $H_{e i}=\operatorname{diag}\{90,90,90\}, \mu_{i 1}(0)=1, \mu_{i 2}(0)=1.5, v_{i 1}=v_{i 2}=100$, and the initial state of the fault estimation observer satisfies $\hat{y}_{e i}(0)=[0.3,1,1.3]^{T}$. When there exists actuator fault, the synchronization of the cyber layer nodes and the physical layer nodes will be broken, and the error $e_{i}$ which is presented in Fig. 7 is biased from zero when compared to the fault free case.
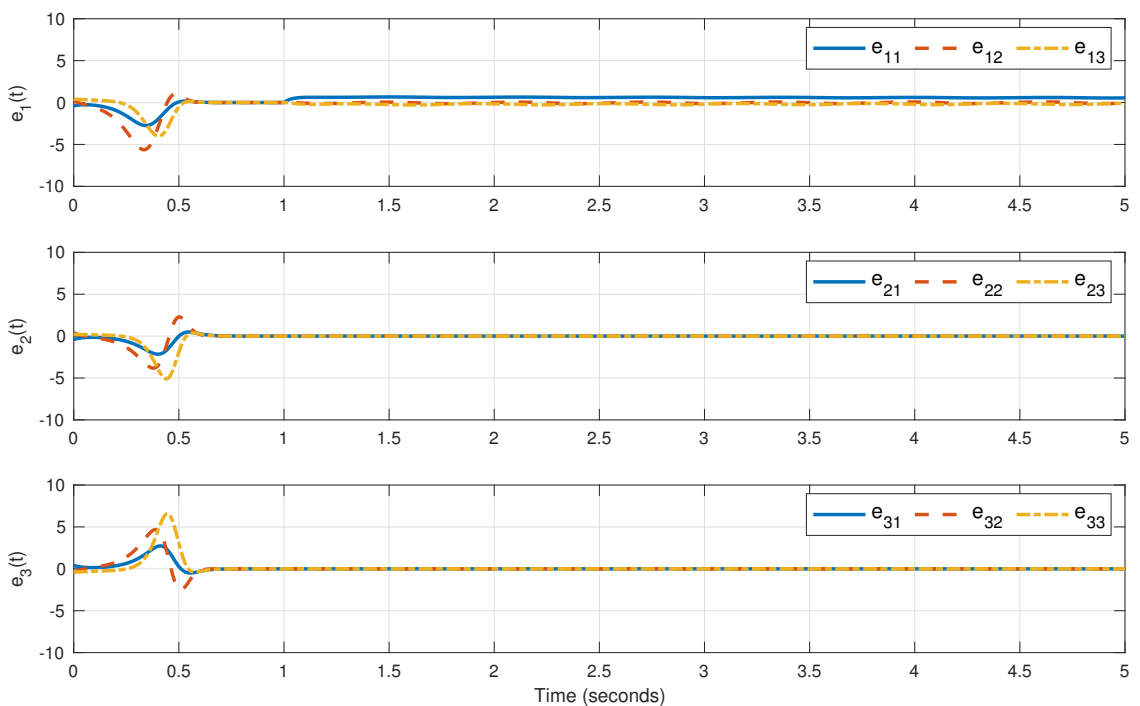

Figure 7: Time responses of $e_{i}$ in actuator single fault case

According to the proposed DFDO, the actuator fault can be detected timely and the related detection curves are depicted in Fig. 8. Once the actuator fault takes place, the corresponding fault detection residual will exceed the adaptive threshold, then the actuator fault can be detected in time. In addition, a fixed threshold fault detection observer proposed in [25] is also implemented in order to make a comparison between the adaptive threshold method and the fixed threshold method. From Fig. 9, it can be noticed that the fixed threshold method indeed can provide a timely fault alarm with respect to the actuator bias fault. However, by referring the time-axises, the bias fault alarm sent by the fixed threshold fault detection observer is triggered a slightly later than the adaptive threshold method in this paper. This merit is owing to the decreasing factor $e^{-\left(\lambda_{\min }\left(Q_{i}\right) / \lambda_{\max }\left(P_{i}\right)\right)\left(t-t_{0}\right)}$ which can provide an exponentially decrease for the time response in the adaptive fault detection threshold. Therefore, the proposed adaptive fault detection observer can provide a faster fault detection and a timely fault alarm when compared to the fixed threshold detection method for the actuator bias fault case. 


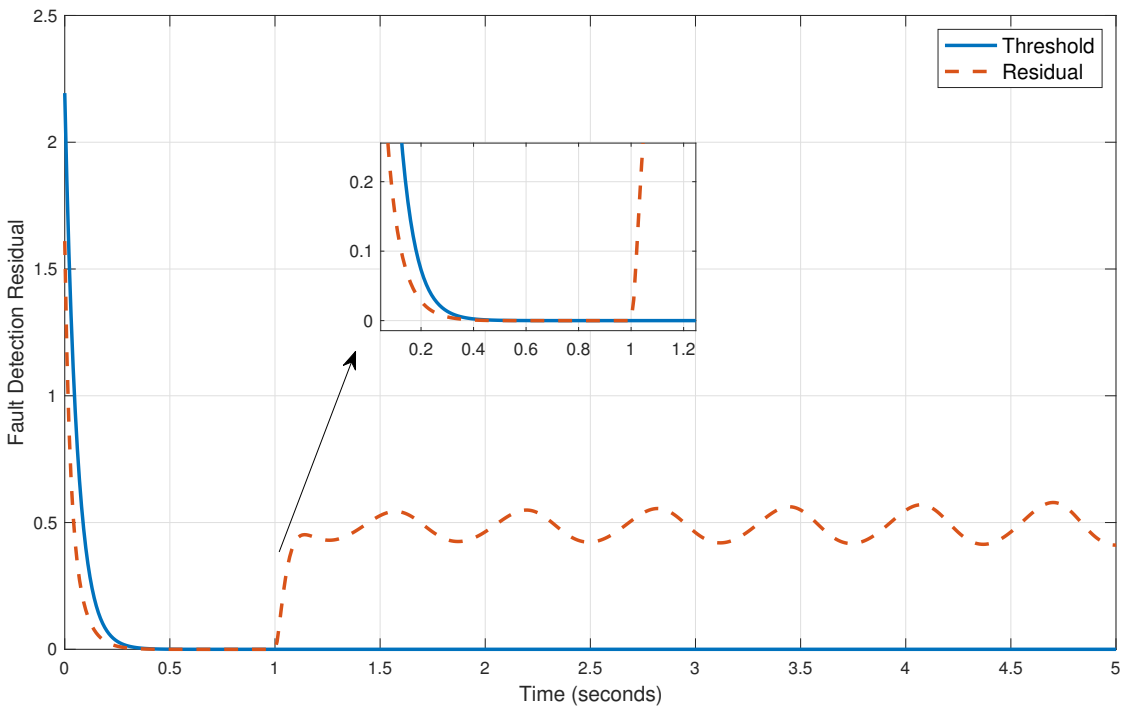

Figure 8: Time responses of the fault detection curves with actuator bias fault

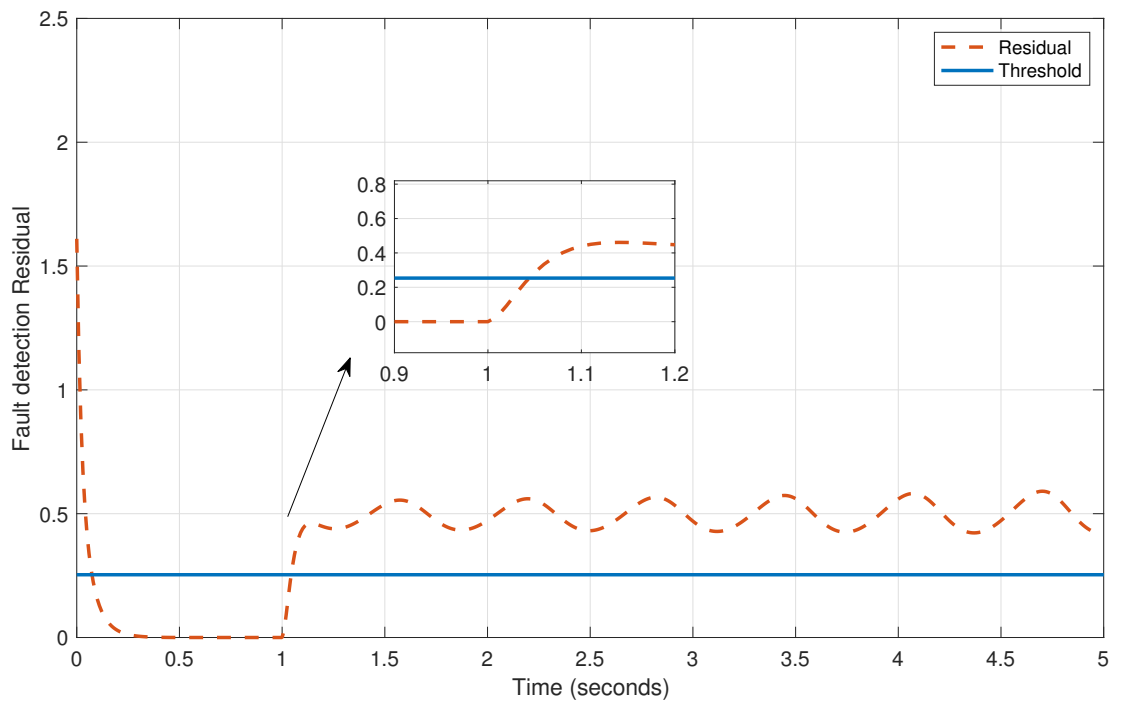

Figure 9: Time responses of the fault detection curves with fixed threshold 
According to the DFEO, the value of actuator bias fault can be reconstructed in Fig. 10. Similarly, a robust fault estimator is also provided in [18]. The estimation provided by the DFEO shows a better estimation process and accuracy with respect to the fault reconstruction method in [18]. In addition, the dynamic estimation process of the bias fault can be modified by $\mu_{i 1}$. It should be noted that the dynamic process of the parameter $\mu_{i 1}$ not only stimulate the fault estimation procedure but also guarantee the estimation error with an asymptotically convergence time response for the actuator constant bias fault while the result from [18] shows an uniformly ultimately bounded performance.

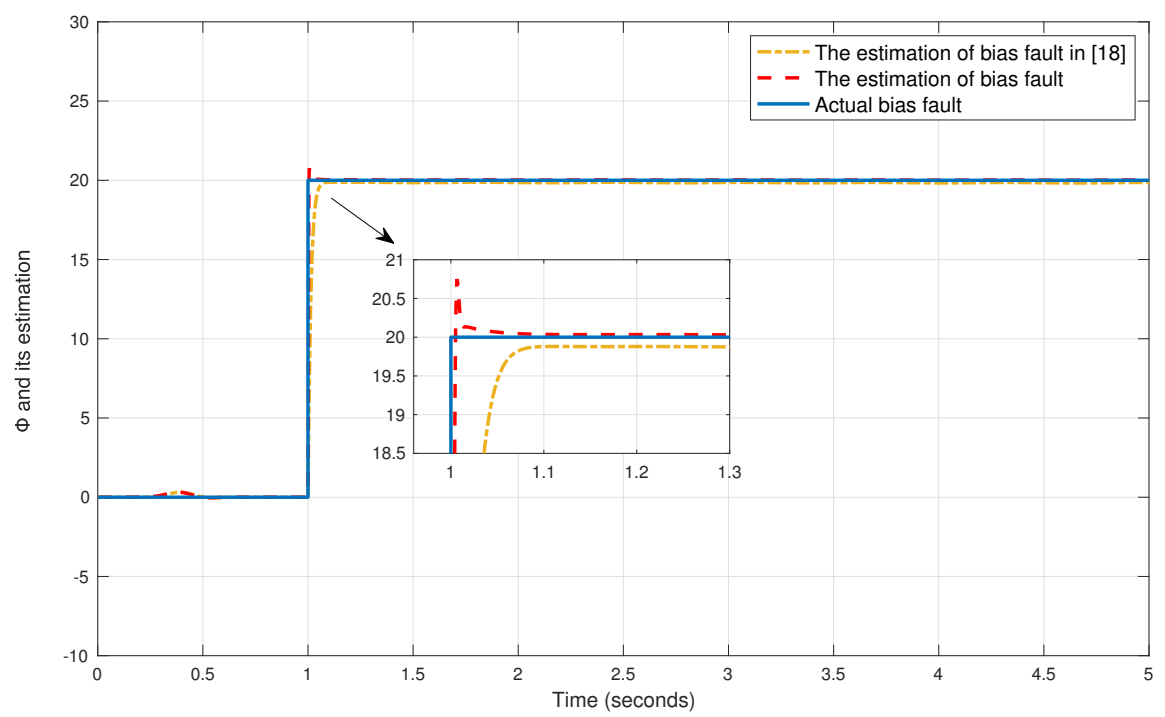

Figure 10: The estimation of actuator bias fault

\subsection{Actuators with multiple types of fault}

In this subsection, it is assumed that the actuators in the physical layer nodes are suffering from two different types of faults, namely, actuator bias fault and LOE fault. Similar to the aforementioned subsection, actuator faults only take place in $y_{1}$. The actuator bias fault and the efficiency factors here are chosen as follows

$$
\begin{aligned}
f_{1} & = \begin{cases}{[0,0,0,0,0]^{T},} & \text { if } 0 \leq t<0.5 s \\
{[15,0,0,0,0]^{T},} & \text { else }\end{cases} \\
\phi_{1} & = \begin{cases}{[1,1,1,1,1]^{T},} & \text { if } 0 \leq t<0.5 s \\
{[1,0.5,1,1,1]^{T},} & \text { else }\end{cases}
\end{aligned}
$$

It can be noticed that the first actuator in the physical layer node $y_{1}$ is suffering from actuator bias fault at $0.5 \mathrm{~s}$ and the second actuator loses $50 \%$ of its control power when the time is $0.5 \mathrm{~s}$. The parameters of the proposed DFDO and DFEO are selected as $P_{i}=\operatorname{diag}\{1.3,1,1.2\}, H_{i}=\operatorname{diag}\{21,22,21\}$, the initial states of the fault detection observer are $\hat{y}_{d i}(0)=[-0.3,0.5,0.3]^{T}$. As for the fault estimation observer, the related parameters are given as $H_{e i}=\operatorname{diag}\{95,92,93\}, \mu_{i 1}(0)=1, \mu_{i 2}(0)=1.6, v_{i 1}=v_{i 2}=120$, and the initial state of the fault estimation observer satisfies $\hat{y}_{e i}(0)=[0.3,1,1.3]^{T}$. The time responses of the error $e_{i}$ is demonstrated in Fig. 11. The error $e_{i}$ is deviated from the desired trajectory curves which is shown in Fig. 4 with respect to the occurrence of the bias fault and LOE fault.

Once the faults take place on the corresponding actuators, the proposed DFDO can detect the occurrence of the actuator faults timely, which is depicted in Fig.12. From Fig.12, it can be noticed that the residual curve crosses 

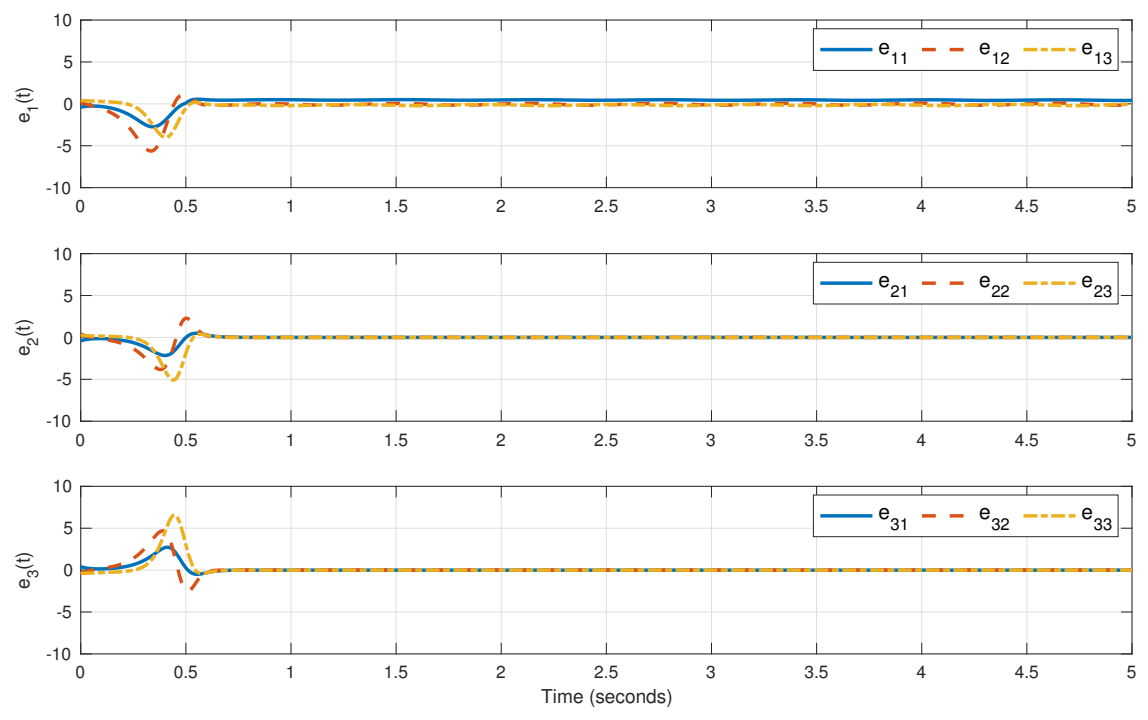

Figure 11: Time responses of $e_{i}$ in actuator multiple fault case

the adaptive threshold determined by the proposed fault detection observer after the occurrence of the actuator faults within a short time. While the fixed threshold method in [25] takes more time to identify the occurrence of the actuator faults. Besides, the fixed threshold based fault detection observer in [25] also demonstrates a slightly insensitivity to the actuator loss of effectiveness scenario in the simulation process when compared to the adaptive threshold method in this paper. This problem which the insensitivity to the actuator LOE fault is alleviated in the proposed adaptive threshold based fault detection scheme and the corresponding theoretical proof is given in Section 4.1.

The estimation of actuator bias fault and the efficiency factor, which are estimated by the proposed DFEO, are shown in Figs 14 and 15. Compared with [24], the estimation of the corresponding efficiency factor has a lower overshoot and a rather smoother estimation process which is very important for the construction of an active fault tolerant controller. This lower overshoot and the rather smoother estimation process is resulted from the adaptive process of the parameter $\mu_{i 1}$. Due to the existence of the $-\mu_{i 1} \hat{\phi}_{i}$ in the adaptive law of $\hat{\phi}_{i}$, the fault estimation procedure is faster than the result presented by the classical adaptive fault estimation observer. Besides, the dynamic tuning $\mu_{i 1}$ changes the time response of $\hat{\phi}_{i}$ when compared to a fixed parameter $\mu_{i 1}$ that can lead to a lower overshoot with respect to the fault estimation result in [24].

Different from [18, 24], the proposed DFEO can provide not only the estimation of actuator bias fault, but also the efficiency factor simultaneously. This advantage may expend the potential application of the proposed adaptive DFEO.

\section{Conclusion}

The robust DFDO and the DFEO for the CPSs' physical layer nodes are proposed in this paper with respect to the existence of the external disturbance. Both actuator bias fault and LOE fault can be detected in time by implementing the widely-used adaptive threshold method. In addition, the proposed DFEO can offer an accurate estimation of the actuator faults by introducing a dynamic process to the corresponding adaptive parameters. The simulation with two different actuator faulty cases demonstrates the effectiveness of the proposed DFDO and the DFEO. However, the fault detection and estimation method in this paper can not deal with the actuator saturation situation, and the solution of fault detection and estimation in actuator saturation case will be our future research work. In addition, the relaxation on the pre-knowledge of the external disturbance in the fault detection observer and fault estimation observer for the cyber-physical systems is another promising direction for the researchers' future work. 


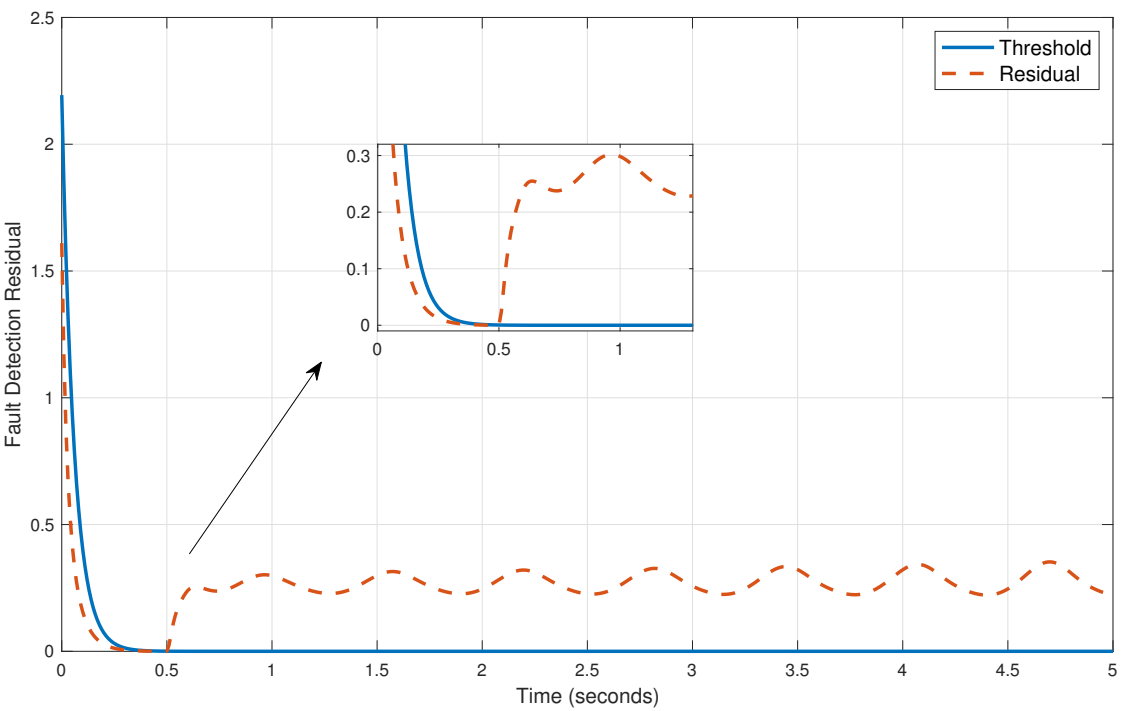

Figure 12: Time responses of the fault detection curves in actuator multiple fault case

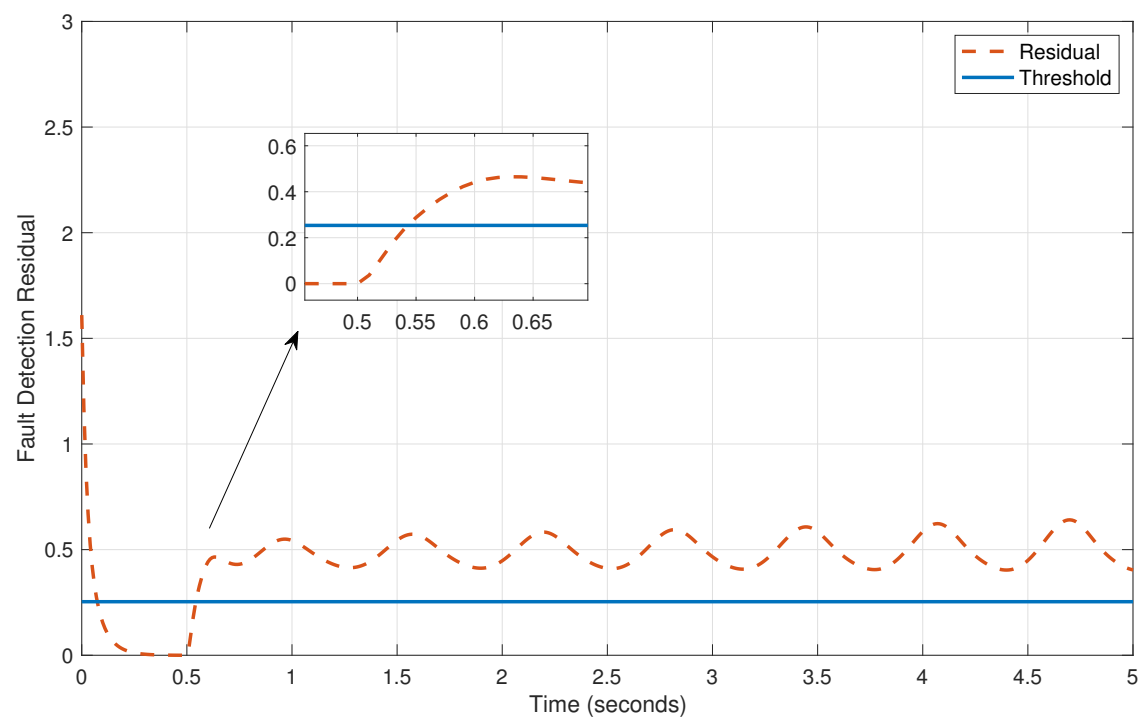

Figure 13: Time responses of the fault detection curves in actuator multiple fault case 


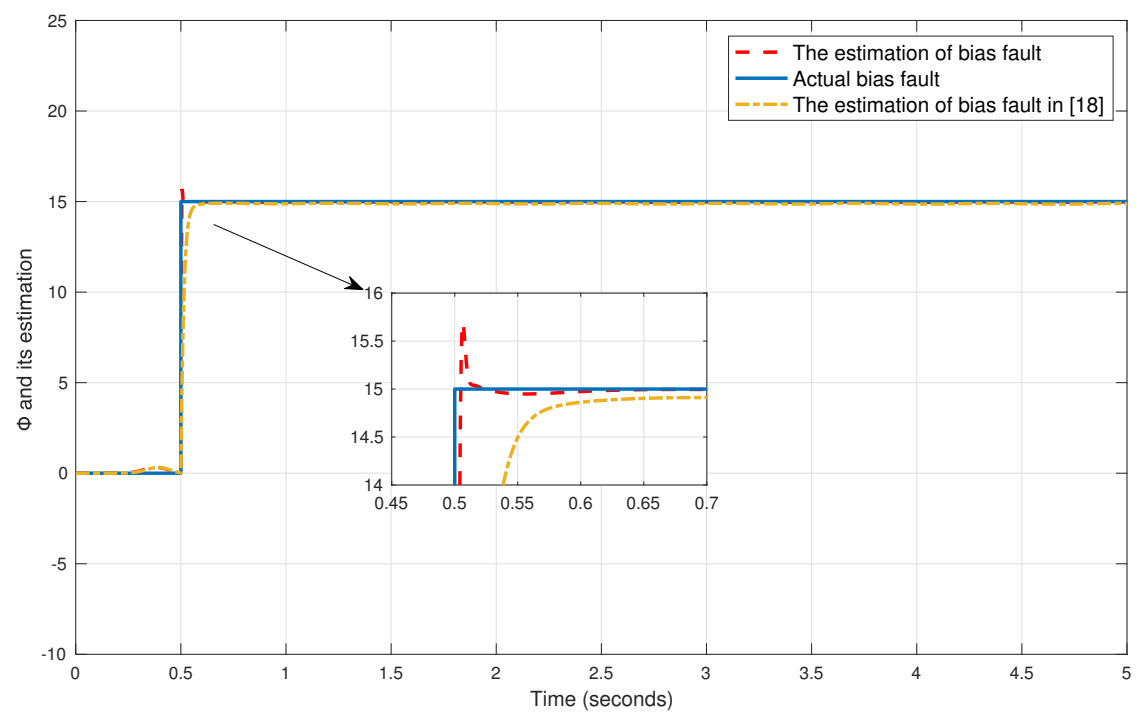

Figure 14: The estimation of actuator bias fault in actuator multiple fault case

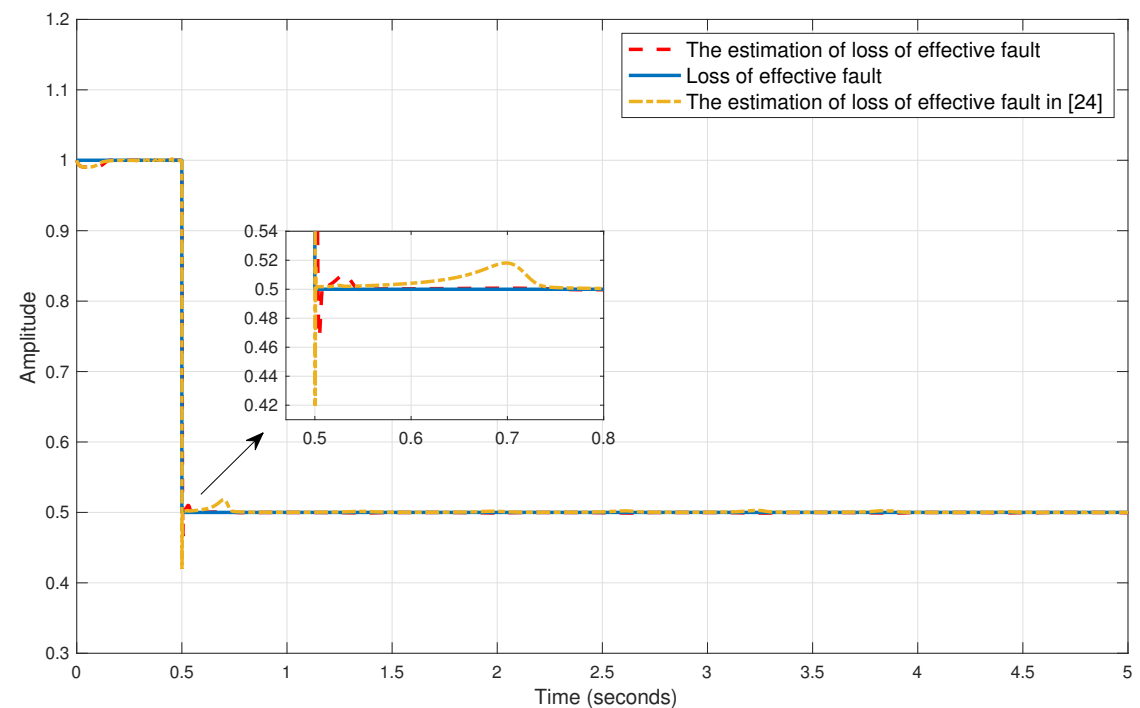

Figure 15: The estimation of corresponding efficiency factor in actuator multiple fault case 


\section{Acknowledgments}

This work was supported by National Natural Science Foundation (NNSF) of China under Grant (No. 61973140, 61973236 and 61573256).

\section{References}

[1] H. Zhang, D. Ge, J. Liu, et al. Multifunctional cyber-physical system testbed based on a source-grid combined scheduling control simulation system. IET Generation Transmission and Distribution, 11(12):3144-3151, 2017.

[2] A. Maffei, S. Srinivasan, D. Meola, et al. A cyber-physical systems approach for implementing the receding horizon optimal power flow in smart grids. IEEE Transactions on Sustainable Computing, 3(2): 98-111, 2018.

[3] M. Housh, Z. Ohar. Model-based approach for cyber-physical attack detection in water distribution systems. Water Research, 139: 132-143, 2018.

[4] P. Antsaklis, B. Goodwine, V. Gupta, et al. Control of cyberphysical systems using passivity and dissipativity based methods. European Journal of Control, 19(5):379-388, 2013.

[5] P. Derler, E. Lee and A. Vincentelli, Modeling cyber-physical systems. In Proceedings of the IEEE, 100(1): 13-28, 2012.

[6] V. Reppa, M. Polycarpou, C. Panayiotou. Distributed sensor fault diagnosis for a network of interconnected cyber-physical systems. IEEE Transactions on Control of Network Systems, 2(1):11-23, 2015.

[7] F. Pasqualetti, F. Dorfler, F. Bullo. Attack detection and identification in cyber-physical systems. IEEE Transactions on Automatic Control, 58(11): 2715-2729, 2013.

[8] A. Teixeira, I. Shames, H. Sandberg, K. Johansson. Distributed fault detection and isolation resilient to network model uncertainties. IEEE Transactions on Cybernetics, 44(11): 2024-2037, 2014.

[9] Y. Quan, W. Chen, Z. Wu and L. Peng. Distributed fault detection for second-order delayed multi-agent systems with adversaries. IEEE Access, 5: 16478-16483, 2017.

[10] L. Zhao, G. Yang. End to end communication rate-based adaptive fault tolerant control of multi-agent systems under unreliable interconnections. Information Sciences, 460-461: 331-345, 2018.

[11] V. Palleti, T. Chong, L. Samavedham. A mechanistic fault detection and isolation approach using Kalman filter to improve the security of cyber physical systems. Journal of Process Control, 68: 160-170, 2018.

[12] X. Zhang, Q. Zhang. Distributed fault diagnosis in a class of interconnected nonlinear uncertain systems. International Journal of Control, 85(11): 1644-1662, 2012.

[13] Y. Jiang, J. Liu, S. Wang. A consensus-based multi-agent approach for estimation in robust fault detection. ISA Transactions, 53(5): 1562$1568,2014$.

[14] X. Li, C. K. Ahn, D. Lu and S. Guo. Robust Simultaneous Fault Estimation and Nonfragile Output Feedback Fault-Tolerant Control for Markovian Jump Systems. IEEE Transactions on Systems, Man, and Cybernetics: Systems. To be published, doi: 10.1109/TSMC.2018.2828123.

[15] P. Menon, C. Edwards. A sliding mode observer for monitoring and fault estimation in a network of dynamical systems. International Journal of Robust and Nonlinear Control, 24(17): 2669-2685, 2014.

[16] D. Ye, S. Luo. A co-design methodology for cyber-physical systems under actuator fault and cyber attack. Journal of The Franklin Instituteengineering and Applied Mathematics, 356(4): 1856-1879, 2019.

[17] X. Yan, C. Edwards. Robust decentralized actuator fault detection and estimation for large-scale systems using a sliding mode observer. International Journal of Control, 81(4): 591-606, 2008.

[18] J. Zhu and G. Yang. Robust distributed fault estimation for a network of dynamical systems. IEEE Transactions on Control of Network Systems, 5(1): 14-22, 2018.

[19] P. Menon, C. Edwards. Robust fault estimation using relative information in linear multi-agent networks. IEEE Transactions on Automatic Control, 59(2), 477-482, 2014

[20] K. Zhang, B. Jiang, P. Shi. Adjustable parameter-based distributed fault estimation observer design for multiagent systems with directed graphs. IEEE Transactions on Cybernetics, 47(2): 306-314, 2017.

[21] R. Alguliyev, Y. Imamverdiyev, L. Sukhostat. Cyber-physical systems and their security issues. Computers in Industry, 100(1): 212-223, 2018.

[22] F. Harirchi, N. Ozay. Guaranteed model-based fault detection in cyber-physical systems: A model invalidation approach. Automatica, 93: 476-488, 2018.

[23] D. Wang, Z. Wang, M. Chen, W. Wang. Distributed optimization for multi-agent systems with constraints set and communication time-delay over a directed graph. Information Sciences, 438: 1-14, 2018.

[24] J. Cai, H. Ferdowsi, J. Sarangapani. Model-based fault detection, estimation, and prediction for a class of linear distributed parameter systems. Automatica, 66: 122-131, 2016.

[25] Z. Gao, B. Jiang, P. Shi, M. Qian, J. Lin. Active fault tolerant control design for reusable launch vehicle using adaptive sliding mode technique. Journal of the Franklin Institute, 349(4): 1543-1560, 2012.

[26] K. Rothenhagen, F. Fuchs. Doubly fed induction generator model-based sensor fault detection and control loop reconfiguration. IEEE Transactions on Industrial Electronics, 56(10):4229-4238, 2009.

[27] G. Chen, Y. Song. Robust fault-tolerant cooperative control of multi-agent systems: A constructive design method. Journal of the Franklin Institute, 352(10), 4045-4066, 2015.

[28] A. Lu, G. Yang. Secure Luenberger-like observers for cyber-physical systems under sparse actuator and sensor attacks. Automatica, 98: 124-129, 2018. 
[29] A. Lu, G. Yang. Secure state estimation for cyber-physical systems under sparse sensor attacks via a switched Luenberger observer. Information Sciences, 417: 454-464, 2017.

[30] B. Huo, Y. Xia, L. Yin and M. Fu. Fuzzy Adaptive Fault-Tolerant Output Feedback Attitude-Tracking Control of Rigid Spacecraft. IEEE Transactions on Systems, Man, and Cybernetics: Systems, 47(8): 1898-1908, 2017. 